\title{
Microstructure and mechanism of adiabatic shear fracture during serrated chip formation of hard machining
}

\author{
Chunzheng DUAN* and Minjie WANG* \\ *School of Mechanical Engineering, Dalian University of Technology \\ Dalian 116024, China \\ E-mail: dcz71@163.com
}

Received 12 December 2013

\begin{abstract}
The formation and development of serrated chip morphology and adiabatic shear bands (ASBs) and the microstructure of fracture surfaces in the serrated chips during hard machining of hardened alloy steel are observed, the mechanism of adiabatic shear fracture is analyzed. The observation and analysis indicate that the cutting speed has significant influence on the transformation of chip morphology and microscopic morphology of ASBs, the material properties can significantly influence on microstructural pattern of fracture surfaces and fracture mechanism during serrated chip formation. The ductile damage fracture induced by adiabatic shear has decisive effect on serrated chip formation, a microcosmic model of adiabatic shear ductile fracture during hard machining including nucleation, growth, coalescence and fracture of microvoids within ASB is proposed.
\end{abstract}

Key words : Hard machining, Adiabatic shear fracture, Microstructure, Serrated chip, Adiabatic shear band

\section{Introduction}

The hard machining is defined as a cutting process of materials with hardness higher than 50 HRC. The application of hard machining technology has been increased drastically during recent years in the manufacturing industry. Precision finish hard machining has the potential to replace grinding in both small and large variety production. A number of experimental and theoretical works regarding various research issues in hard machining such as chip morphology(Guo and Yen, 2004), cutting forces(Huang and Liang, 2005), tool wear (Singh and Rao, 2007), surface roughness (Grzesik and Wanat, 2005) have been reported. The understanding of chip formation plays an important role in machining process optimization and surface integrity. There are obvious differences in chip formation mechanism between hard machining and common machining. As cutting speed increases, a serrated chip, which has significant influence on cutting force, cutting temperature, tool wear and quality of machined surface, frequently is generated during machining hardened materials. The serrated chips often look like discontinuous chips formed during machining materials with lower hardness under low cutting speed, in fact, the mechanisms of two chip types are quite different, the formation mechanism of serrated chip therefore has received much attention. Different mechanisms for serrated chip formation have been proposed by many researchers in the past few decades, up to now, there is no accordant viewpoint on understanding reason for serrated chip formation. In general, the formation reason for serrated chip is not only not the machine-tool vibration, but not the stick slip instability in second shear zone during material removing. The investigations under different cutting conditions and experiment materials showed that the shear instability fracture in primary shear zone (PSZ) is a main reason for serrated chip formation in steady state cutting condition with good machine-tool stiffness. As the research on chip formation in hard machining is increasingly thorough, two theory systems on formation mechanism of serrated chip gradually have formed, which are the adiabatic shear theory based on heat transfer proposed by Komanduri (1982) and Komanduri et al.(1982) and the periodic fracture theory based on the fracture mechanics proposed by Nakayama et al.(1988). Komanduri is a precursor to suggest that serrated chip formation can be explained using adiabatic shear theory, Komanduri and co-workers observed the chip formation processes of hardened high strength alloy steel and Titanium alloy using high speed photography and put forward a model with two stages for serrated chip formation. After this, Davies et al.(1997) also presented a model of serrated 
chip formation to support the adiabatic shear theory. In recent years, many scholars have investigated the serrated chip formation processes through quick-stop tests and metallurgical observations of chip samples for various materials including titanium and its alloys (Gente and Hoffmesiter, 2001; Molinari et al., 2002; Komanduri and Hou, 2002; Wan et al., 2012; Ye et al., 2013), Nickel base alloy(Zhang et al., 2008), stainless steel (Manyindo and Oxley, 1986) and quenched high strength steel (Barry and Byrne, 2002; Duan et al., 2009; Morehead et al., 2007; Li et al., 2012), the results showed the good agreement between most of experimental phenomena and adiabatic shear model, they agreed with that the adiabatic shear bands(ASBs) observed by microscopy is a strongest evidence for supporting adiabatic shear theory. After successful explaining the mechanism of serrated chip formation using adiabatic shear theory, in the contrary, some scholars found out a number of microcracks near PSZ during quick-stop tests for carburizing steel (Shaw and Vyas, 1998; Vyas and Shaw, 1999)and hardened high strength steel (Nakayama et al., 1988), therefore, they made an objection to the model of serrated chip formation process based on adiabatic shear theory and suggested that the root reason leading to serrated chip formation is not ductile fracture induced by adiabatic shear, but brittle fracture along PSZ. Nakayama et al. (1988) presented a model with periodic fracture for serrated chip formation, Shaw and Vyas (1998) and Vyas and Shaw (1999) first proposed a periodic fracture theory to explain serrated chip formation mechanism, they thought that the serrated chips are caused by periodic integral fracture occurring after the microcracks extend to half distance from the free surface to the cutting edge, the shear angle of fracture plane is assumed to be $45^{0}$. Elbestawi et al.(1996) presented a criterion for crack initiation and propagation during the high speed machining of hardened steel based on fracture mechanics, and in-depth studied the periodic brittle fracture mechanism. Koenig et al. (1993) also proposed a similar theory for turning hardened steel and explained the serrated chip formation mechanism based on hypothesis of shear stress expansion.

So far, the controversy still continues, however, either of theory models must be attributed to the fracture mechanism within PSZ, therefore, it is necessary to clarify microstructure pattern of fracture surfaces inside serrated chips. The material failure process from instability to fracture within PSZ has a significant influence on chip morphology, cutting force fluctuation, tool wear mechanism and machined surface quality. According to the material fracture theory under high strain rate loading, the fracture mechanism for cutting process, especially for high speed cutting, can not be explained only using ductile fracture caused by thermal instability or brittle fracture based on macroscopic fracture mechanics. In general, a number of defects such as microcracks and microvoids exist in engineering materials, during high speed deformation, the accumulation, expansion and connection of damages promoted by adiabatic temperature rise often occur inside ASBs that should be an essential reason leading to final failure, therefore, the fracture criterion during cutting process may be not based on ideal macroscopic fracture mechanics, but based on damage fracture mechanics. In order to determine the theoretical basis for model of serrated chip formation and give an insight into its formation mechanism, it is needed to observe the formation and development of chip morphology and ASB and the microstructure of fracture surface of serrated chip and to reveal the microscopic process from damage to fracture inside serrated chip.

To explore the fracture mechanism during serrated chip formation, in this paper, the formation and development of serrated chip morphology and ASBs and the microstructure pattern of fracture surfaces in the serrated chips during hard machining of alloy steel with two hardnesses were observed, the micro-fracture mechanism inside the serrated chips and the influence of hardness on fracture were analyzed.

\section{Experimental procedure}

High strength low alloy steel of 30CrNi3MoV with composition (in wt.\%) of 0.30\% C, 0.90\% Cr, 3.15\% Ni, 0.30\% Mo, $0.20 \% \mathrm{~V}, 0.27 \% \mathrm{Si}, 0.45 \% \mathrm{Mn}$ and balance Fe, was used in this study. The experimental materials were machined into disc specimens, $95 \mathrm{~mm}$ in diameter and $3.5 \mathrm{~mm}$ in height. The specimens were quenched from $890^{\circ} \mathrm{C}$ and then tempered at $200^{\circ} \mathrm{C}$ and $500^{\circ} \mathrm{C}$ for 1 hour, the final hardness values are HRC55 and HRC50 respectively. The orthogonal cutting experiments were carried out on a high-speed lathe with cutting speed ( $v$ ) range from 20 to 500 $\mathrm{m} / \mathrm{min}$, cutting depth of $0.215 \mathrm{~mm}$, cutting width of $3.5 \mathrm{~mm}$, and tool rake angle of $-10^{\circ}$.

The serrated chips were collected, and then the cross sections and free surfaces of the chip specimens were selected as observed surface for optical microscope and SEM. The chips first were embedded in the bakelite, after sanding, polishing and eroding, the microstructure of the primary shear zone (PSZ) was examined by optical microscope, and the microstructure pattern of fracture surface was observed by SEM. 


\section{Results and discussion}

\subsection{Formation and development of serrated chip morphology and ASBs}

Fig. 1 is the metallographic photos of typical chip morphology formed in the cutting process accompanied by the emergence and development of ASBs. Fig.2 shows three kinds of microscopic morphology of ASBs as the cutting speed increases during serrated chip formation. As the cutting speed changes from low to high, the chip morphology transforms from ribbon chip(Fig.1(a)) into serrated chip with ASBs (Fig.1(b),(c)) and serrated chip with fracture (Fig.1(d)). The previous researches found that, during the cutting process of hardened steel, two main types of ASB were produced which are deformed band and transformed band. There were the severely broken martensite laths like dynamic recovery microstructure within the deformed bands, however, inside the transformed bands, a large number of equiaxed grains with very small diameter were found, the dynamic recrystallization and phase transformation took place (Duan et al., 2009; Duan and Zhang, 2012). In this study, the serrated chips with deformed bands and transformed bands also are observed. In Fig.2(a), the deformed shear band has larger width in which the microstructure is elongated along the shear band direction. Since the temperature rise in deformed band is insufficient for inducing grain refinement and phase transformation, the grains only are elongated and broken along the shear band, therefore, the contrast between deformed band and its surrounding is not clear, as shown in Fig.1(b) and Fig.2(a). With increase of cutting speed, the shear deformation localization becomes more intense, the deformation is highly concentrated in the shear bands and the temperature also quickly increases that lead to very narrow transformed bands. Due to the occurrence of recrystallization or phase transformation, the corrosion resistance of transformed band advances, they appear as "white and bright band", as shown in Fig.1(c) and Fig.2(b). When the cutting speed continues to increase, the adiabatic shear further develops, the cracks propagate along the ASB until go throughout the whole chip, the fracture occurs under the tensile stress finally, as shown in Fig.1(d) and Fig.2(c). In Fig.2(c), it is worth while to note that a crack propagates from the free surface to the bottom of chip along the ASB and is located close to the boundary of ASB, the crack tip is passivated showing the thermal effect during crack formation. Because of the obvious gradients of temperature and strain at the junction of ASB and chip matrix where the microvoids and microcracks are easy to be initiated and to grow up, therefore, the fracture often occurs at the boundaries of ASBs.

The observations of ASBs show that after the initiation of ASBs, along with cutting conditions change, the ASBs will show different types, the cutting speed has significant influence on transformation of microscopic morphology of ASBs. This indicates a fact that the occurrence of adiabatic shear fracture contains a series of rate dependent processes including emerging and developing of deformed band, transforming from deformed band into transformed band, developing of transformed band, propagating of cracks along transformed band leading to fracture.
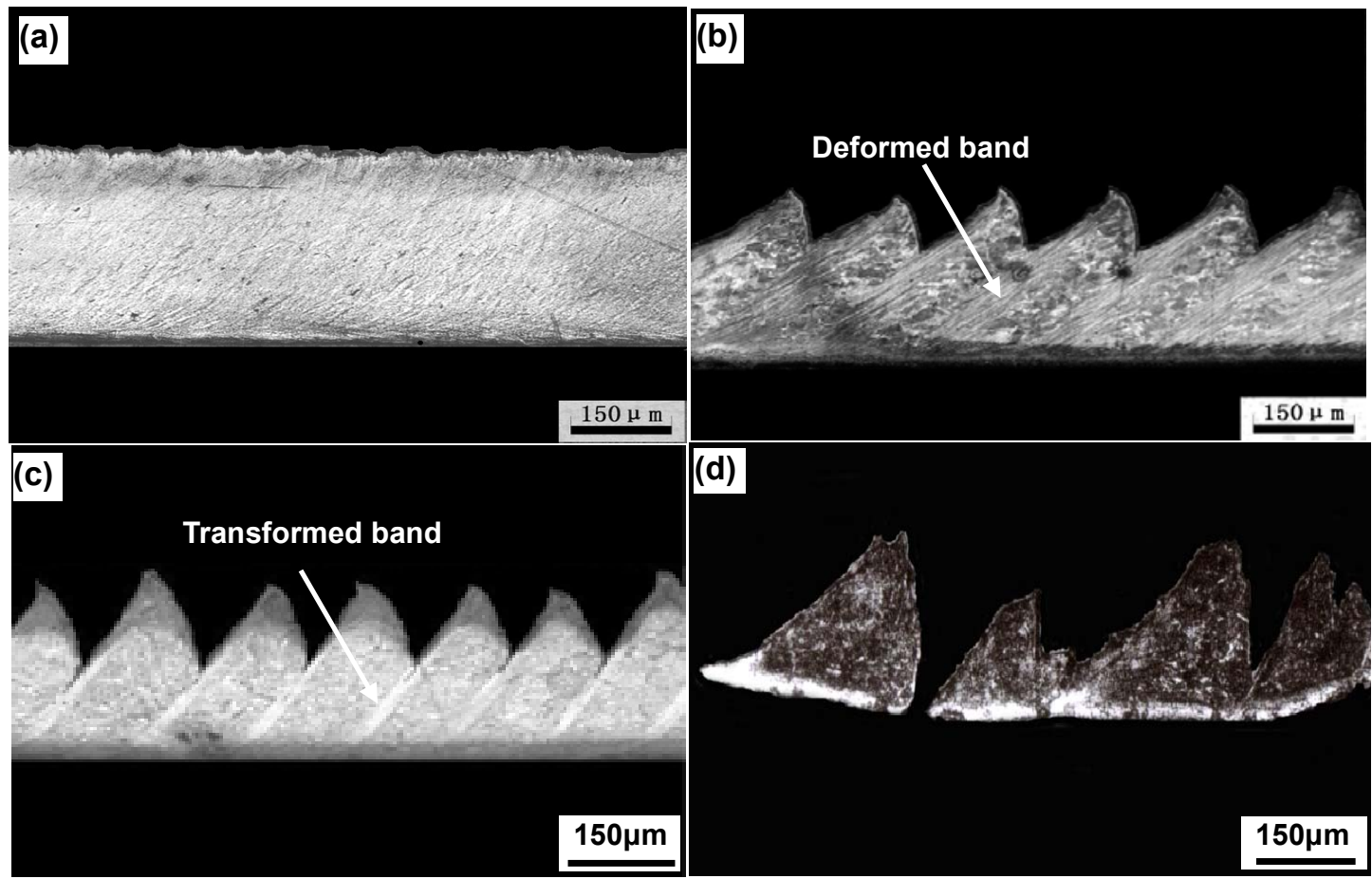

Fig.1 Formation and development of serrated chip morphology and ASBs with cutting speed (material hardness: HRC55) (a) ribbon chip $(v=40 \mathrm{~m} / \mathrm{min})$, (b) serrated chip with deformed bands $(v=90 \mathrm{~m} / \mathrm{min})$, (c) serrated chip with transformed bands $(v=180 \mathrm{~m} / \mathrm{min})$, (d) serrated chip with fracture $(v=500 \mathrm{~m} / \mathrm{min})$ 


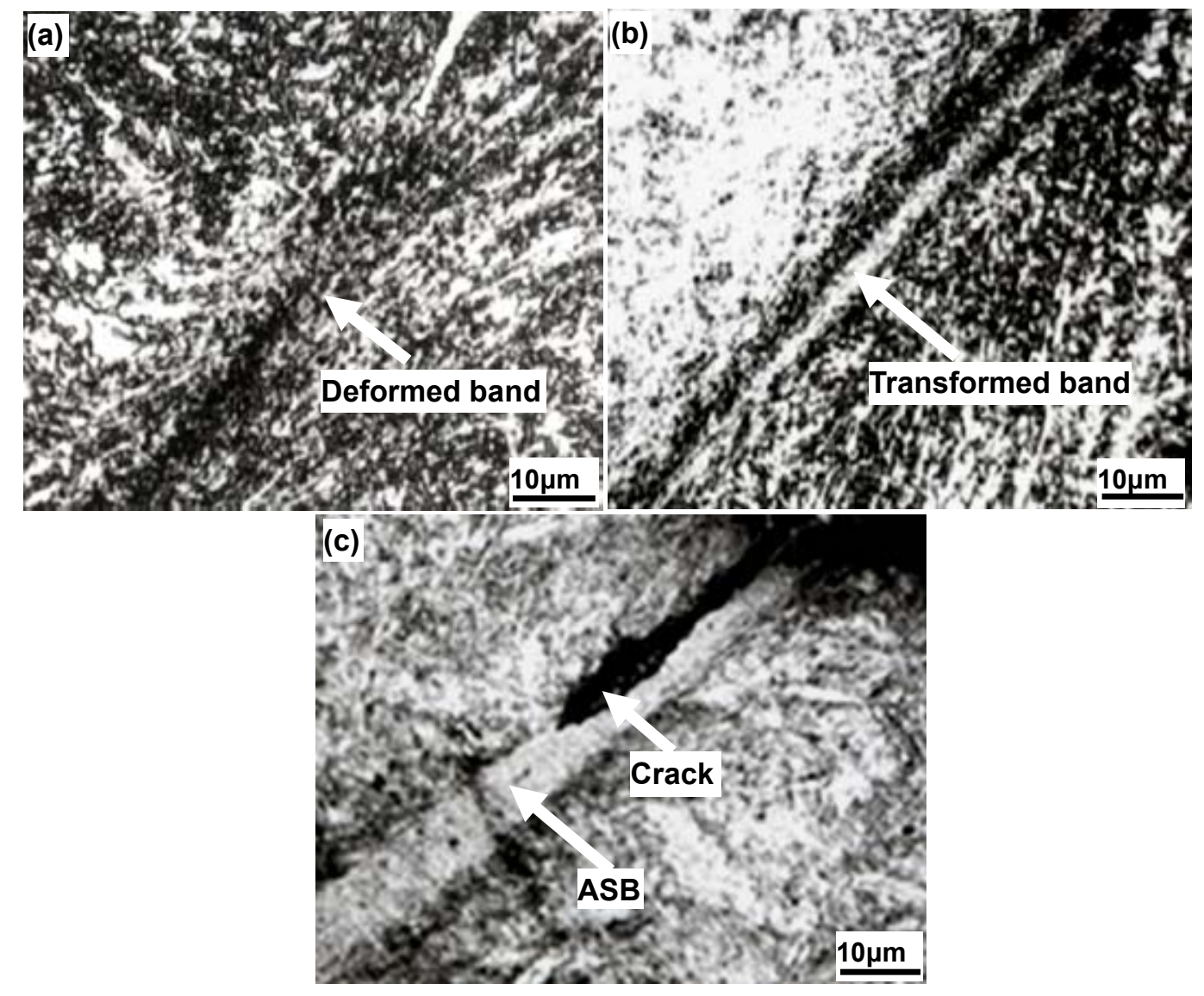

Fig.2 Three kinds of microscopic morphology formed during development of the ASBs (material hardness: HRC55) (a) deformed shear band ( $v=90 \mathrm{~m} / \mathrm{min})$, (b) transformed shear band $(v=150 \mathrm{~m} / \mathrm{min})$, (c) transformed shear band with cracks $(v=180 \mathrm{~m} / \mathrm{min})$

\subsection{Microstructure observation of fracture surface in serrated chip}

Fig. 3 is the micrographs of fracture surfaces of a serrated chip with transformed bands for high temperature tempered steel (hardness: HRC50). Fig.3(a) shows the morphology of a serrated chip and the microscopic observation direction vertical to cross-section. The low magnification observation reveals that the fracture surfaces are divided into two distinct $\mathbf{A}$ and $\mathbf{B}$ areas, the area $\mathbf{A}$ is larger and rough, but the area $\mathbf{B}$ is flat, as shown in Fig.3(b). The high magnification micrograph of area $\mathbf{A}$ in Fig.3(c) shows a number of dimples which are elongated along the shear direction and look like parabola shape. Fig.3(d) is a high magnification micrograph of area $\mathbf{B}$ showing that this area is not smooth and includes a lot of knobble structures between which there are many micro-pits and small flat surfaces that seem to form after melting. The microcracks are not found, most of fracture surfaces are covered with the elongated dimples, so these fracture surfaces have an apparent shear ductile fracture characteristic. Thus, for high temperature tempered steel (hardness: HRC50), it is clear that the fracture inside PSZ of serrated chip is not caused by periodic brittle fracture, the reason for fracture only can be explained by occurrence and development of adiabatic shear instability under the action of adiabatic temperature rise. The numerous experiment results showed that one of the most significant morphology characteristics of fracture surface in ASBs of steels is strongly elongated dimple microstructure which is produced under shearing stress when ASBs just are completed (Wittman and Meyers, 1990; Cho et al., 1990; Giovanola et al., 1998). Thereby, the formation of serrated chip is induced by adiabatic shear fracture. With regard to the knobble structures in the area $\mathbf{B}$, some similar observation results were reported by Bedford et al. (1974) under shock compression fracture for high carbon steel, and by Cho et al.(1990) and Giovanola et al.(1998) under torsion loading for high strength steel. The formation of knobble structures contains a series of fracture and friction processes including adiabatic shear fracture caused by growth and coalescence of microvoids, severe friction between a relatively 
large area pairs of fracture surface, thermal fluctuation and even partial melting produced by friction making the ligaments between elongated dimples disorientated and disappeared, and final producing flat knobble structures (Rogers, 1979).

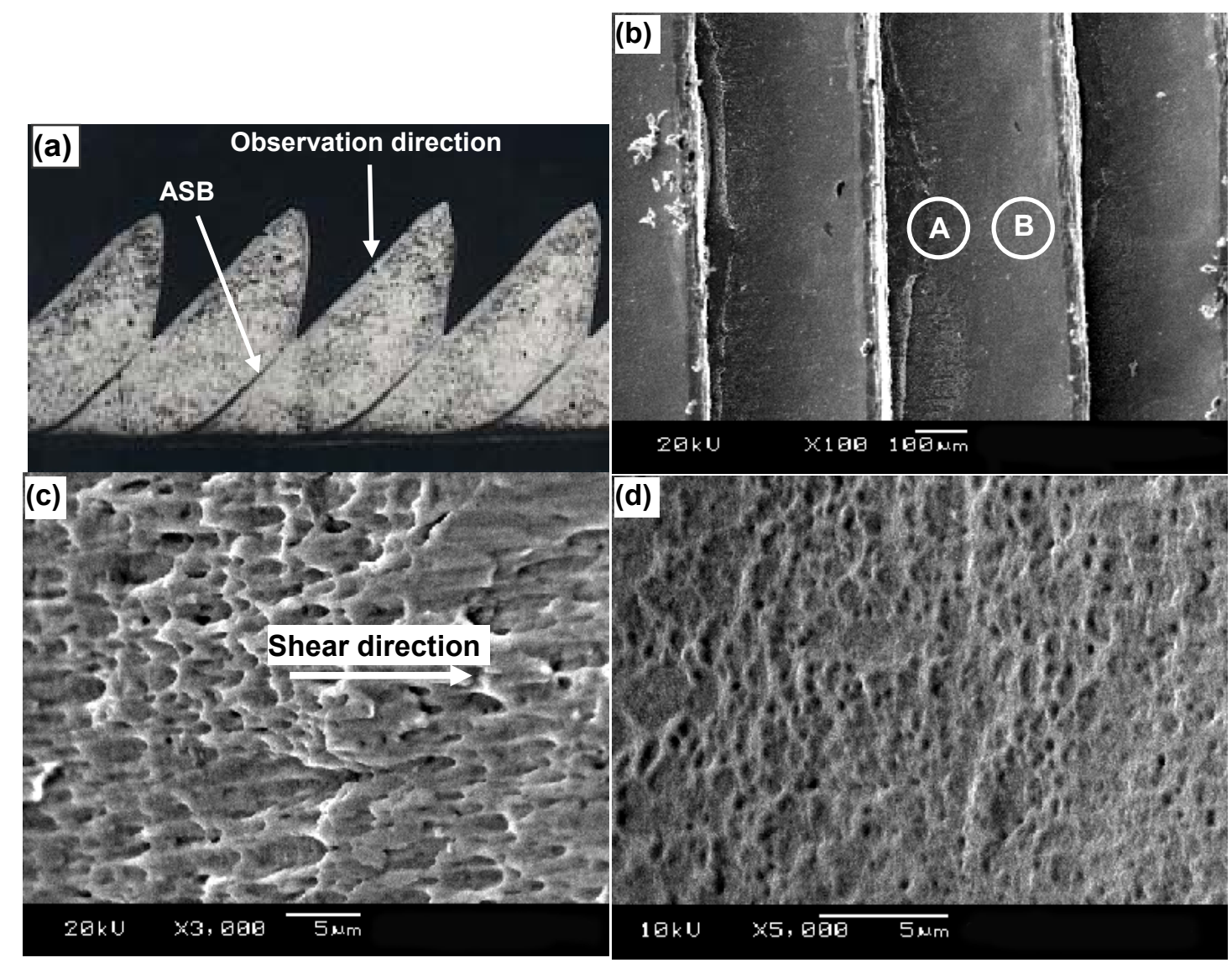

Fig.3 SEM micrographs of fracture surfaces of a serrated chip with transformed bands (material hardness: HRC50, $v=300$ $\mathrm{m} / \mathrm{min}$ ) (a) morphology of a serrated chip, (b) a lower magnification micrograph showing the whole fracture surfaces, (c) a higher magnification micrograph of $\mathbf{A}$ area showing the elongated dimple structures along the shear direction, (d) a higher magnification micrograph of $\mathbf{B}$ area showing the knobble structures due to heavy rubbing between fracture surfaces

Fig.4 is the micrographs of fracture surfaces of a serrated chip with transformed bands for low temperature tempered steel (hardness: HRC55). Fig.4 (a) shows the morphology of a serrated chip, its sawtooth degree is greater than that of high temperature tempered steel (hardness: HRC50) at same cutting speed, while the microscopic pattern of fracture surfaces is different from high temperature tempered steel (see Fig.3). A lower magnification micrograph shows that the whole fracture surface is divided into three areas, the area $\mathbf{A}$ is a rough surface, the area $\mathbf{B}$ is bright and flat, the area $\mathbf{C}$ is dark and flat, see Fig.4(b). An amplified photo of area $\mathbf{A}$ in Fig.4(c) shows the elongated dimple structures along the shear direction which is similar to that in Fig.3(c) for lower hardness, but the fine knobble structures are not found. The micrograph of $\mathbf{B}$ area shows a large number of tiny voids connected by microcracks, as shown in Fig.4(d). In the $\mathbf{C}$ area, the lamellar structures containing a large number of slip stripe distributed in the shear direction are observed as shown in Fig.4(e).

Fig.5 is the micrographs of dimple areas on fracture surfaces of serrated chips with deformed bands, it is found from Fig. 5 that, compared with fracture surfaces of serrated chips with transformed bands in Fig. 3 and Fig.4, not only the number of dimples decreases obviously but also their depth reduces, the fracture surfaces become more flat, these characteristics indicate that because the deformed bands are formed under low cutting speed, the damage degree in the shear bands is smaller that leads to the decrease in number of microvoids, which are difficult to grow up due to lower temperature rise, so the more flat dimple areas on fracture surfaces are observed. In addition, the number of dimples for HRC55 hardness material is more than that for HRC50 hardness material, this indicates that the material properties have significant influence on the fracture pattern in the deformed bands. 

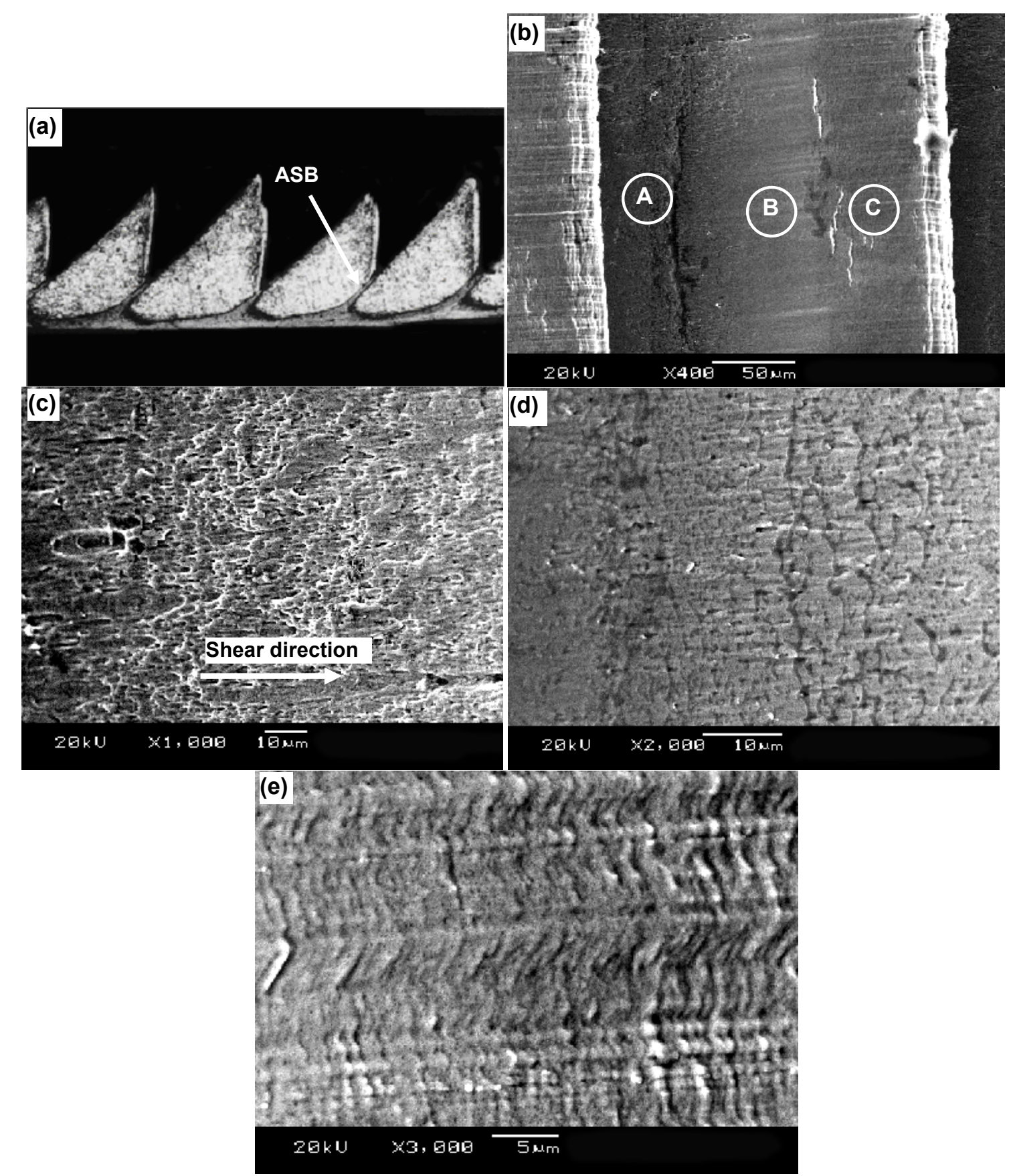

Fig.4 SEM micrographs of fracture surfaces of a serrated chip with transformed bands (material hardness: HRC55, v=300 $\mathrm{m} / \mathrm{min}$ ). (a) morphology of serrated chip, (b) a lower magnification micrograph showing the whole fracture surfaces, (c) a micrograph of $\mathbf{A}$ area showing the elongated dimple structures, (d) a higher magnification micrograph of $\mathbf{B}$ area showing the microcracks and microvoids, (e) a higher magnification micrograph of $\mathbf{C}$ area showing the lamellar structures

The observations of fracture surfaces in the serrated chips show the difference in microscopic pattern of fracture surfaces between materials with two hardness values, this indicates that the material properties can significantly influence on fracture mechanism during serrated chip formation. For low temperature tempered steel with higher hardness, the $\mathbf{B}$ and $\mathbf{C}$ areas in front of the dimple area obviously have no thermal softening characteristics, so these areas should be produced before the adiabatic shear occurs, a number of microcracks and small voids indicate that the microstructure damage already is very strong before the formation and fracture of ASBs. Komanduri and Brown (1981) described in detail the microcracks in shear zone during metal cutting process and thought that the instability in PSZ is caused by material microstructure heterogeneity, the microcracks easily are generated at dislocations, grain boundaries, microvoids, second phase particles and other defects. In low temperature tempered $30 \mathrm{CrNi3MoV}$ steel, the size of 
carbide particles is larger, even in the absence of a high temperature rise, the microvoids are prone to be generated and connected into microcracks. Under the large compressive stress at the tip of cutting tool, the microcracks can be welded, the fracture is inhibited. While the compressive stress at free surface is very small, due to material microstructure heterogeneity, the local tensile stress may even appear during high speed deformation that causes microcracks to extend to the bottom of chips, as shown in Fig.2(c). The observations of fracture surfaces in the serrated chips with two tempering hardnesses indicate that although there are differences in microscopic pattern, the ductile fracture induced by adiabatic shear has decisive effect on serrated chip formation, while the microstructure damage occurring before adiabatic shear instability can accelerate fracture process.
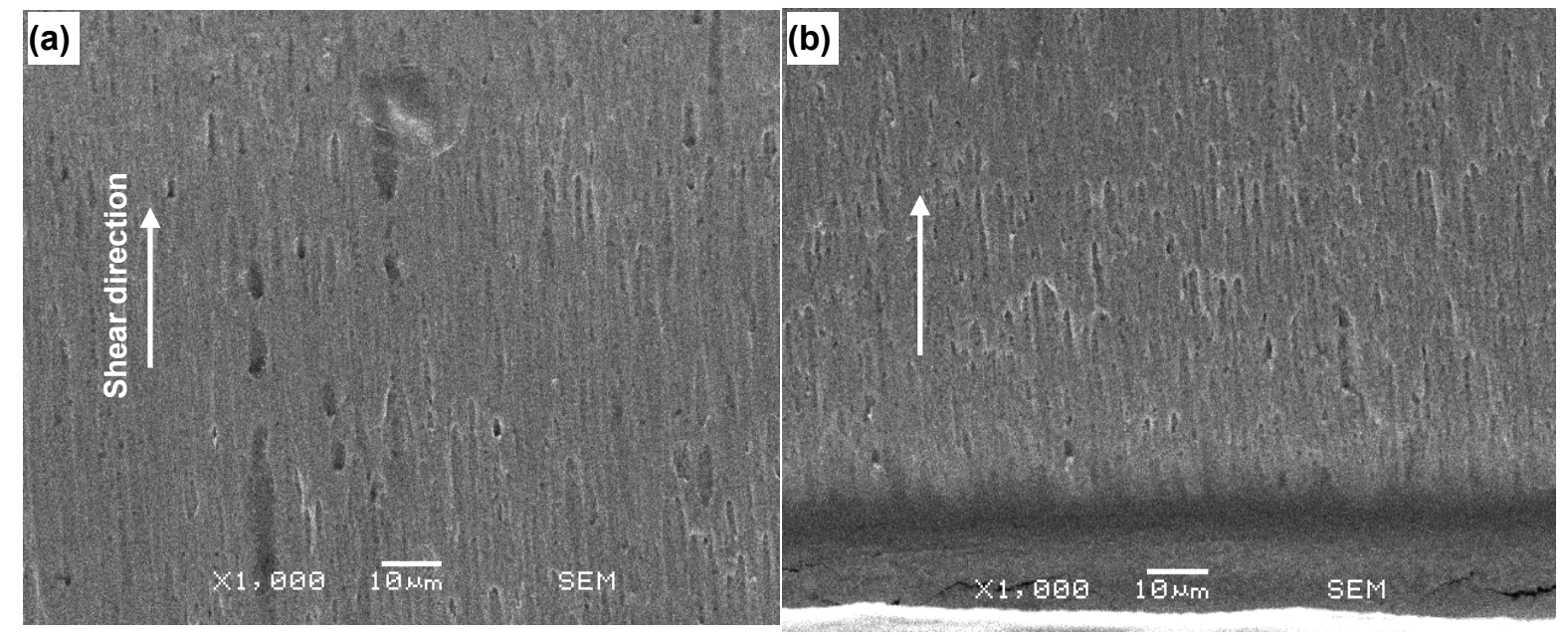

Fig.5 SEM micrographs of dimple areas on fracture surfaces of serrated chips with deformed bands ( $\mathrm{v}=90 \mathrm{~m} / \mathrm{min})$

(a) material hardness: HRC50, (b) material hardness: HRC55

\subsection{Microscopic modelling and mechanism analysis of adiabatic shear fracture during hard machining}

It is found through above observations that adiabatic shearing fracture is a damage accumulation process. It is already clear that ASB is a micro-damage mode which is induced under the action of compressive stress, shear stress or combined compression and shear stress, the formation of ASBs is accompanied by accumulation of microvoids which grow up and then connect each other to form macroscopic cracks resulting in final fracture along ASBs. Fig. 6 is the orthogonal cutting model of serrated chip formation. In this model, there are the shear stress $\left(F_{S}\right)$ parallel to shear plane and the normal stress $\left(F_{n}\right)$ perpendicular to shear plane in PSZ during cutting process, the material in PSZ is at combined compression and shear stress state and so can meet the condition triggering adiabatic shear. With the increase of cutting speed, the thermal softening in PSZ is gradually increased, at a certain critical cutting condition, the thermal softening exceeds strain hardening, ASBs will be produced in PSZ if the adiabatic shear instability condition is met. The research showed that (Giovanola, 1998) the stress state can strongly influence the formation and fracture of ASBs, the high compressive stress is beneficial to the formation of ASB leading to that ASBs are often initiated at site where the maximum compressive stress is achieved. During cutting process, the distribution of compressive and shear stresses is not uniform along length direction of PSZ, the normal stress reaches a maximal value at contact point of PSZ and tool tip and then decreases, while the shear stress remains unchanged in finite length followed by decreasing slowly. Therefore, ASBs should first originate from tool tip and then extend to free surface. The triggering ASB at tool tip is also related to the following factors: (1) shear angle decreases near tool tip causing very high strain and strain rate here; (2) friction between chip bottom and rake tool face provides additional heat source for occurrence of adiabatic shear instability leading to higher temperature near tool tip than that at other positions. These analysises are consistent with theoretical model proposed by Komanduri and Brown (1981) and validated by quick-stop tests. The tool tip is the site of ASB initiation, but the fracture is developed from the free surface of chips to inside. The high compression stress at tool tip can restrain the formation of microvoids, the compression stress at free surface is minimum that is easy for nucleation and growth of microvoids, consequently the fracture often starts at the free surface.

The damage fracture not only can be a brittle fracture mode by forming and developing of microcracks at microstructure nonuniform sites such as inclusions, second phase particles or interface defects, but also can occur by a 
ductile fracture mode controlled by nucleating, growing and coalescing of microvoids inside ASB, this is dependent on stress state and deformation history. If when adiabatic shear fracture is triggered, shear band is still in thermal softening state, the fracture will occur by ductile fracture mode. According to the viewpoint of Wittman and Meyers(1990), the microvoids should rapidly nucleate and grow at defects after adiabatic shear occurs as well as before ASBs are not cooled under the action of high temperature, the microcracks initiated at defects will be expanded and passivated rapidly to form elliptical microvoid cores. These can demonstrate that the ductile fracture occurs at early stage of adiabatic shear instability process.

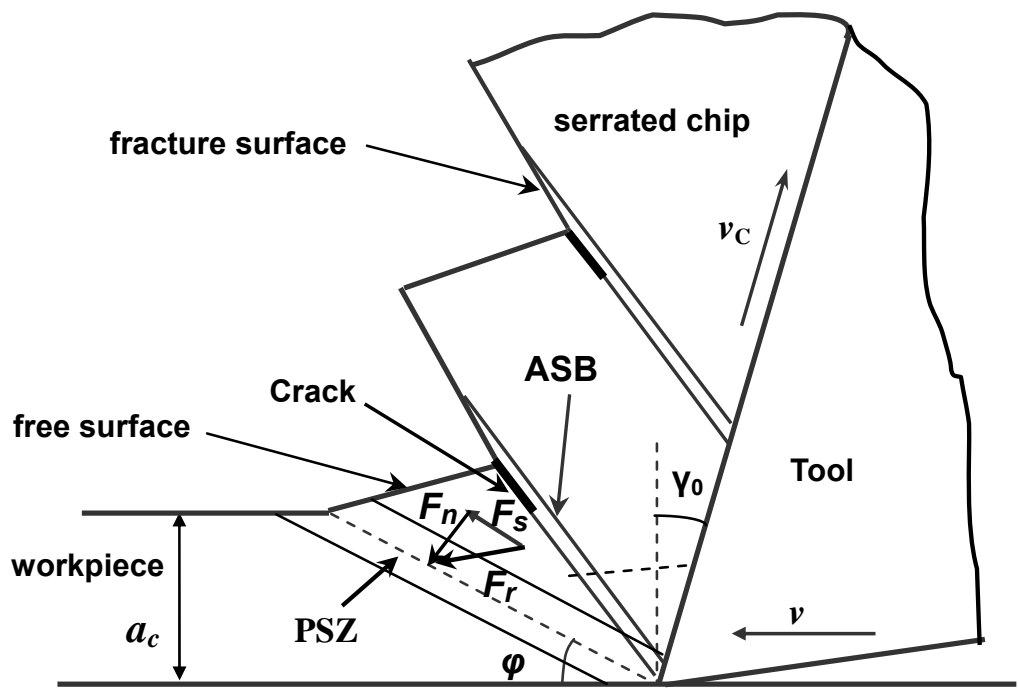

Fig.6 Orthogonal cutting model of serrated chip formation

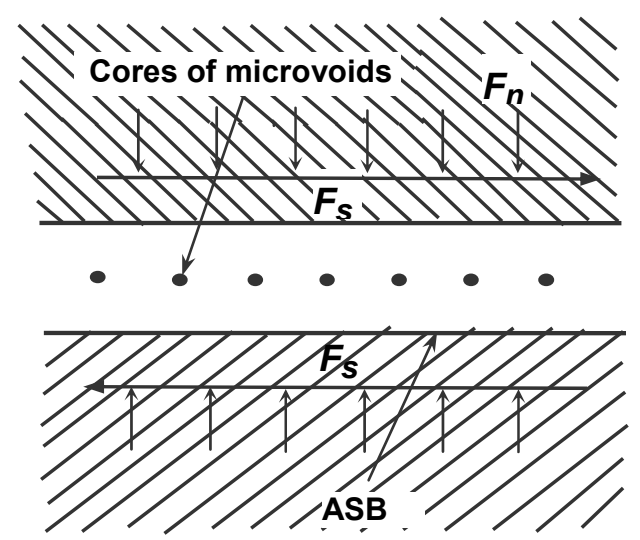

(a)
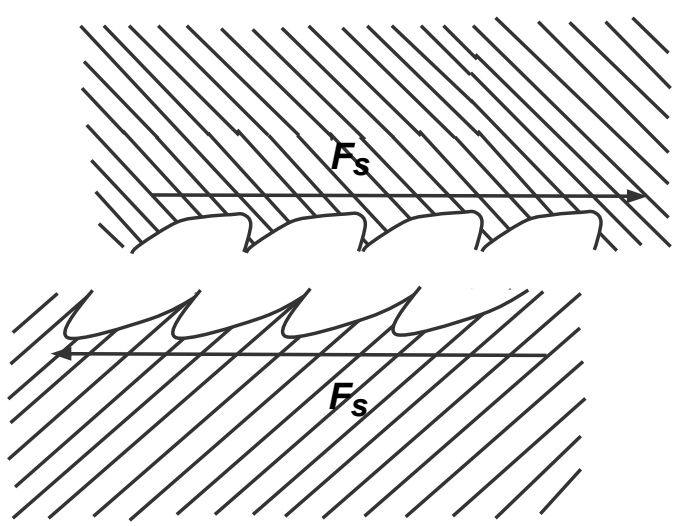

(c)

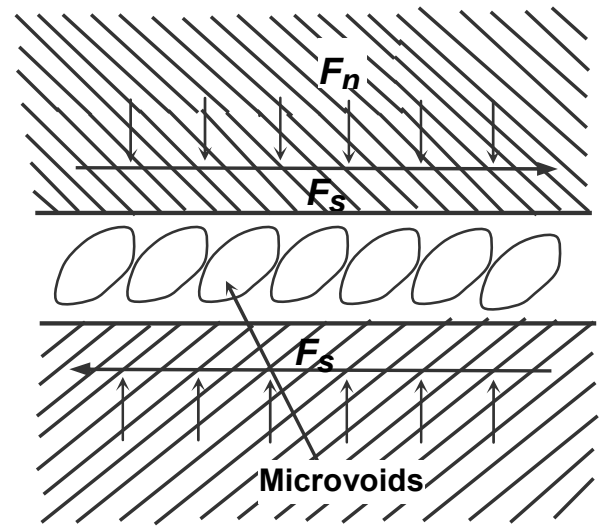

(b)
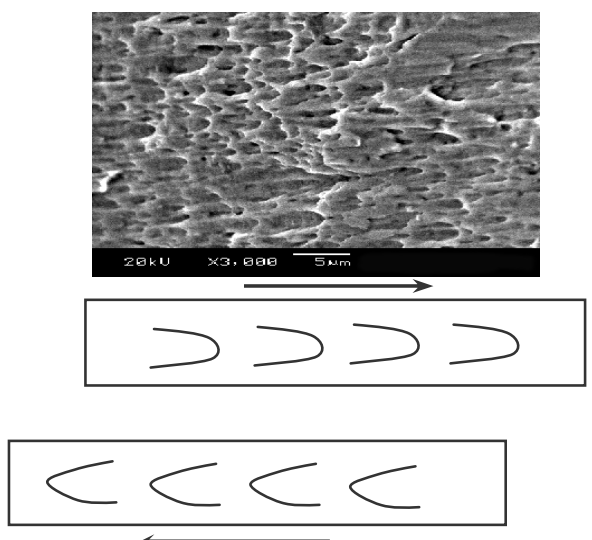

(d)

Fig.7 Microcosmic model of adiabatic shear ductile fracture process occurring in ASB through nucleation,growth and coalescence of microvoids. (a) nucleation of microvoids in ASB, (b) growth and coalescence of microvoids along shear direction, (c) fracture of coalesced microvoids under shear stress, (d) elongated dimple structures along shear direction on adiabatic shear fracture surfaces 
On the basis of the results of fracture surface observations and fracture mechanism analysis, a microscopic model of ductile fracture process occurring inside ASB can be built up to illustrate a series of process including nucleating, growing and coalescing of microvoids until fracture, as shown in Fig.7. In hardened steels, the dispersed and fine particles of alloy carbide often become the nucleation points of microvoids. At the initial stage of adiabatic shear, the deformation incompatibility between hard carbide particle and metallic matrix can cause cracking of combination surface, and then a large number of very small cores of microvoid appear within ASB (Fig.7(a)). At this stage, the material in ASB is in a composite state of compression and shear stresses, and the temperature is not high, therefore, the microvoids can not grow rapidly. As the temperature in ASB increases, the microvoids begin to grow up, coalesce and elongate along shear direction under the shear stress (Fig.7(b)). Some macroscopic cracks appear after the microvoids interconnect, then the adiabatic shear fracture occurs along ASB and finally the elongated dimple structures remain on fracture surface, as shown in Fig.3(c), Fig.4(c), Fig.(5), Fig.7(c) and Fig.7(d). A series of microcosmic fracture processes are accomplished instantaneously, so the real time observation during experiment is very difficult. Therefore, this microcosmic fracture model presented in Fig.7 will need more experiments to confirm.

The observations in this study show that the differences in microstructure properties between experimental materials with two hardnesses result in different microscopic pattern of fracture surfaces, this indicates that the microstructure homogeneity and hardness of materials can significantly affect microcosmic fracture process. It is worth noting that the elongated dimple structures are found on both the fracture surfaces, therefore, the final reason leading to the formation of serrated chips still is a ductile adiabatic shear fracture for present experiment materials. Since the high temperature tempered material has good microstructure homogeneity and toughness, there is no severe microstructure damage before adiabatic shear instability, the fracture occurs in thermal softening state all the time and is governed by formation and development of microvoids. For the low temperature tempered material, a large number of microcracks are produced due to microstructure damage before initiating adiabatic shear, so the fracture surfaces show some brittle fracture characteristics, this fracture process may be regarded as a mixed mode of ductile adiabatic shear fracture and brittle periodic fracture. Adiabatic shear instability and fracture is two processes that are competitive and independent. Adiabatic shear instability is affected by the adiabatic stress-strain performance and can be modeled through continuum mechanics. But the fracture process is accompanied by strength decrease and energy dissipation, this stage is composed of nucleation, growth and coalescence of microvoids, must be described by a statistical model of micro-damage.

Based on the above microscopic model of adiabatic shear damage fracture, the adiabatic shear damage evolution equations can be established as follows. In a volume of finite element $V$, there are $n$ microvoids with mean volume $v$ and radius $R$. If $S$ represents the surface area of each microvoid, the damage degree $D$ is defined as the ratio between total volume of microvoids and element volume, $\dot{D}$ represents the damage evolution rate, the following equations are got:

$$
\begin{aligned}
& s=4 \pi R^{2}, v=\frac{4}{3} \pi R^{3}, \dot{v}=\frac{R \dot{s}}{2} \\
& D=\frac{n v}{V}=\frac{4 / 3 n \pi R^{3}}{V}, \quad v=\frac{D V}{n}, \quad R=\sqrt[3]{\frac{3 D V}{4 \pi n}} \\
& \dot{D}=\frac{n \dot{v}}{V}-\frac{n v \dot{V}}{V^{2}}=\frac{n \dot{v}}{V}-\frac{n v \theta}{V}
\end{aligned}
$$

In Eq. (3), the nucleation effect is ignored. $\theta=\dot{V} / V$ represents the relative volume expansion rate. The surface energy per unit area of microvoid is expressed as $\lambda$, according to the principle of energy conservation during damage evolution, the following equation is obtained:

$$
n \lambda \dot{s}=\chi V \dot{W}_{V}^{I}
$$


Where $\dot{W}_{V}^{I}$ is the plastic power per unit volume, $\chi$ is the scale factor transforming plastic work into surface energy during void damage development. From Eqs. (1), (4)

$$
\dot{v}=\frac{R}{2} \dot{s}=\frac{R}{2} \frac{\dot{\chi} V}{n \lambda} \dot{W}_{V}^{I}
$$

If the threshold condition of microvoid growth is determined by the equivalent plastic strain $\bar{\varepsilon}^{p}$, substituting Eqs.

(2) ,(5) into Eq.(3):

$$
\dot{D}= \begin{cases}\frac{\chi \dot{W}_{V}^{I}}{2 \lambda}\left(\frac{3 D}{4 \pi n_{v}}\right)^{1 / 3}-D \theta, & \bar{\varepsilon}^{p} \geq \bar{\varepsilon}_{D}^{p} \\ 0, & \bar{\varepsilon}^{p}<\bar{\varepsilon}_{D}^{p}\end{cases}
$$

Where $n_{v}=n / V$ represents the equivalent activation microvoid number per unit volume, $\bar{\varepsilon}_{D}^{p}$ is the equivalent plastic strain threshold of damage development, when $\bar{\varepsilon}^{p} \geq \bar{\varepsilon}_{D}^{p}$, the damage will develop. Therefore, the Eq. (6) can be the damage evolution equation of microvoids in adiabatic shear band. At present, no mature statistical micro-damage model is proposed, a lot of theoretical and experimental works is still in progressing.

\section{Conclusions}

According to observations of the formation and development of serrated chip morphology and ASB $_{s}$ and the microstructure pattern of fracture surfaces in the serrated chips during machining of hardened 30CrNi3MoV alloy steel, and the micro-fracture mechanism inside the serrated chips and the influence of hardness on fracture are analyzed, the following conclusions are obtained:

(1) As the cutting speed rises, the chip morphology transforms from ribbon chip into serrated chip with ASBs and serrated chip with fracture, three kinds of microscopic morphology of ASBs during serrated chip formation are found, i.e. deformed band, transformed band and transformed band with cracks. The cutting speed has significant influence on the transformation of chip morphology and microscopic morphology of ASBs.

(2) The fracture surfaces in the serrated chips for high temperature tempered steel with lower hardness are divided into two distinct areas, i.e. the area with elongated dimple structures along shear direction and the area with knobble structures, while the fracture surfaces for low temperature tempered steel with higher hardness are divided into three areas including the area with elongated dimple structures, the area with microcracks and microvoids and the area with lamellar structures. The observations indicate that the material properties can significantly influence on microscopic pattern of fracture surfaces and fracture mechanism during serrated chip formation.

(3) The analysis of microscopic fracture mechanism indicates that for the high temperature tempered material with lower hardness, there is no severe microstructure damage before adiabatic shear instability, the formation of serrated chip is based on a ductile fracture process governed by formation and development of microvoids. For the low temperature tempered material with higher hardness, due to microstructure damage before initiating adiabatic shear, this fracture process may be regarded as a mixed mode of ductile adiabatic shear fracture and brittle periodic fracture. The ductile fracture induced by adiabatic shear has decisive effect on serrated chip formation, meanwhile the microstructure damage can accelerate fracture process.

(4) A microcosmic model of adiabatic shear ductile fracture during hard machining within ASB including nucleation, growth, coalescence and facture of microvoids is proposed. 


\section{Acknowledgments}

This research is supported by National Natural Science Foundation of China (Grant No. 51375072, 51175063).

\section{References}

Barry, J., Byrne, G., The mechanisms of chip formation in machining hardened steels, Journal of Manufacturing Science and Engineering, Transactions of the ASME, Vol.124, No.3(2002), pp.528-535.

Bedford, A.J., Wingrove, A.L., Thompson, K.R.L., Phenomenon of adiabatic shear deformation, Journal of the Australian Institute of Metals, Vol.19, No.1(1974), pp.61-73.

Cho, K., Chi, Y.C., Duffy, J., Microscopic observations of adiabatic shear bands in three different steels, Metallurgical Transactions A, Vol.20, No.5(1990), pp.1161-75.

Davies, M.A., Burns, T.J., Evans, C.J., On the dynamics of chip formation in machining hard metals, CIRP Annals Manufacturing Technology, Vol.46, No.1(1997), pp.25-30.

Duan, C.Z., Cai, Y.J., Wang, M.J., Microstructural study of adiabatic shear bands formed in serrated chips during high-speed machining of hardened steel, Journal of Materials Science, Vol.44, No.3(2009), pp.897-902.

Duan, C.Z., Zhang, L.C., Adiabatic shear banding in AISI 1045 steel during high speed machining: Mechanisms of microstructural evolution, Materials Science and Engineering A, Vol.532 (2012), pp. 111-119.

Elbestawi, M.A., Srivastava, A.K., El-Wardany, T.I., Model for chip formation during machining of hardened steel, CIRP Annals - Manufacturing Technology, Vol.45, No.1(1996), pp.71-76.

Gente, A., Hoffmesiter, H.W., Chip formation in machining Ti6AI4V at extremely high cutting speeds, CIRP Annals - Manufacturing Technology, Vol.50, No.1(2001), pp.49-52.

Giovanola, J.H., Adiabatic shear banding under pure shear loading. II. Fractographic and metallographic observations, Mechanics of Materials, Vol.7, No.1(1998), pp.73-87.

Grzesik, W., Wanat, T., Comparative assessment of surface roughness produced by hard machining with mixed ceramic tools including 2D and 3D analysis, Journal of Materials Processing Technology, Vol.169, No.3(2005), pp.364-371.

Guo, Y.B., Yen, D.W., A FEM study on mechanisms of discontinuous chip formation in hard machining, Journal of Materials Processing Technology, Vol.155-156, No.1-3(2004), pp.1350-1356.

Huang,Y., Liang, S.Y., Modeling of cutting forces under hard turning conditions considering tool wear effect, Journal of Manufacturing Science and Engineering, Transactions of the ASME, Vol.127, No.2(2005), pp.262-270.

Koenig, W., Berktold, A., Koch, K.F., Turning versus grinding - a comparison of surface integrity aspects and attainable accuracies, CIRP Annals - Manufacturing Technology, Vol.42, No.1(1993),pp.39-43.

Komanduri, R., Some clarifications on the mechanics of chip formation when machining titanium alloys, Wear, Vol.76, No.1(1982), pp. 15-34.

Komanduri, R., Brown, R.H., On the mechanics of chip segmentation in machining, Journal of Engineering for Industry, Transactions of the ASME, Vol.103(1981), pp.33-51.

Komanduri, R., Hou,Z.B., On thermoplastic shear instability in the machining of a titanium alloy (Ti-6A1-4V), Metallurgical and Materials Transactions A, Vol.32, No.9(2002), pp.2995-3010.

Komanduri, R., Schroeder, T., Hazra, J., Von-Turkovich, B.F., Flom, D.G., On the catastrophic shear instability in high-speed machining of an AISI 4340 steel, Journal of Engineering for Industry, Transactions of the ASME, Vol. 104(1982), pp.121-31.

Li, G.H., Yan, B., Cai, Y.J., Experiment study of chip formation and cutting force of hardened AISI 1045 steel in high speed machining, Advanced Materials Research, Vol.383-390(2012), pp.1915-1920.

Manyindo, B.M., Oxley, P.L.B., Modelling the catastrophic shear type of chip when machining stainless steel, Proceedings of the Institution of Mechanical Engineers, Part C Mechanical engineering scienc, Vol.200, No.5(1986), pp.349-58.

Molinari, A., Musquar, C., Sutter, G., Adiabatic shear banding in high speed machining of Ti-6Al-4V: experiments and modeling, International Journal of Plasticity, Vol.18, No.4( 2002), pp.443-459.

Morehead, M.D., Huang, Y., Luo, J., Chip morphology characterization and modeling in machining hardened 52100 steels, Machining Science and Technology, Vol.11, No.3(2007), pp. 335-54. 
Nakayama, K., Arai, M., Kanda, I., Machining characteristics of hardened matreials, CIRP Annals - Manufacturing Technology, Vol.37, No.1(1988), pp.89-92.

Rogers, H.C., $\quad$ Adiabatic plastic deformation, Annual Review of Materials Science, Vol.9(1979), pp.283-311.

Shaw, M.C., Vyas, A., Mechanism of chip formation with hard turning steel, CIRP Annals - Manufacturing Technology, Vol.47, No.1(1998), pp.77-82.

Singh, D., Rao, P.V., A surface roughness prediction model for hard turning process, International Journal of Advanced Manufacturing Technology, Vol.32, No.11-12( 2007), pp.1115-1124.

Vyas, A., Shaw, M.C., Mechanics of saw-tooth chip formation in metal cutting, Journal of Manufacturing Science and Engineering, Transactions of the ASME, Vol.121, No.2(1999), pp.163-72.

Wan, Z.P., Zhu, Y.E., Liu, H.W., Tang, Y., Microstructure evolution of adiabatic shear bands and mechanisms of saw-tooth chip formation in machining Ti6Al4V, Materials Science and Engineering A, Vol.531(2012), pp.155-63.

Wittman, C.L., Meyers, M.A., Observation of an adiabatic shear band in AISI 4340 steel by high-voltage transmission electron microscopy, Metallurgical Transactions A, Vol.21, No.3(1990), pp.707-716.

Ye, G.G., Xue, S.F., Jiang, M.Q, Tong, X.H., Dai, L.H., $\quad$ Modeling periodic adiabatic shear band evolution during high speed machining Ti-6Al-4V alloy, International Journal of Plasticity, Vol.40(2013), pp. 39-55.

Zhang, D.J., Wang, C., Liu., Chen, M., A FEM and experiment study on high speed machining of nickel-based superalloy GH4169, Key Engineering Materials, Vol.375-376(2008), pp. 82-86. 